\title{
TRANSPARENT CONDUCTING OXIDES. TEXTURE AND MICROSTRUCTURE EFFECTS ON CHARGE CARRIER MOBILITY IN MOCVD- DERIVED CdO THIN FILMS GROWN WITH A THERMALLY STABLE, LOW-MELTING PRECURSOR
}

Andrew W. Metz, John R. Ireland", Jian-Guo Zheng", Ricardo P.S.M. Lobo", Yu Yang, Jun Ni, Charlotte L. Stern, Vinayak P. Dravid", Nicole Bontemps", Carl R. Kannewurf", Kenneth R. Poeppelmeier, and Tobin J. Marks*

Department of Chemistry, Department of Electrical and Computer Engineering ${ }^{\dagger}$, Department of Materials Science and Engineering ${ }^{\ddagger}$, and the Materials Research Center, Northwestern University, Evanston, IL 60208-3113, USA, Laboratoire de Physique du Solide”, Ecole Supérieure de Physique et Chimie Industrielles de la Ville de Paris, CNRS UPR 5, 75321 Paris Cedex 5, France

*To whom correspondence should be addressed. E-mail: t-marks@northwestern.edu. 
Table S1. Crystallographic data for complexes 1 and 4 .

Table s2. Crystal data and structure refinement for 1.

Table S3. Atomic coordinates and equivalent isotropic displacement parameters $\left(A^{\wedge} 2 \times 10^{\wedge} 3\right)$ for 1 . $U(e q)$ is defined as one third of the trace of the orthogonalized Uij tensor.

Table S4. Bond lengths [A] and angles [deg] for 1.

Table S5. Anisotropic displacement parameters ( $\left.A^{\wedge} 2\right)$ for 1.

The anisotropic displacement factor exponent takes the form: $-2 \mathrm{pi}^{\wedge} 2$ [ h^2 a*^2 U11 + .. + $2 \mathrm{~h} \mathrm{k} a * b * \mathrm{U} 12$ ]

Table s6. Hydrogen bond distances [A] and angles [deg] for 1.

Table S7. Crystal data and structure refinement for compound 4.

Table S8. Atomic coordinates and equivalent isotropic displacement parameters $\left(A^{\wedge} 2 \times 10^{\wedge} 3\right)$ for 4 . $U(e q)$ is defined as one third of the trace of the orthogonalized Uij tensor.

Table s9. Bond lengths $[A]$ and angles [deg] for 4.

Table s10. Anisotropic displacement parameters $\left(A^{\wedge} 2\right)$ for 4.

The anisotropic displacement factor exponent takes the form: $-2 \mathrm{pi}^{\wedge} 2\left[\mathrm{~h}^{\wedge} 2 \mathrm{a}{ }^{\wedge} 2 \mathrm{U} 11+\ldots+2 \mathrm{~h} \mathrm{k} a * \mathrm{~b} * \mathrm{U} 12\right]$

Figure S1. Packing diagram for 4. Non-hydrogen bonded hydrogen and disordered fluorine atoms are ommited for clarity.

Figure S2. Variable-temperature ${ }^{1} \mathrm{H}$ NMR of complex 2 (A) and complex 4 (B) in toluene- $d_{8}$ at $500 \mathrm{MHz}$. * Denote solvent resonances. 
Table S1. Crystallographic data for complexes 1 and $\mathbf{4}$

\begin{tabular}{|c|c|c|}
\hline & 1 & 4 \\
\hline empirical formula & $\mathrm{CdC}_{16} \mathrm{H}_{18} \mathrm{O}_{6} \mathrm{~F}_{12} \mathrm{~N}_{2}$ & $\mathrm{CdC}_{18} \mathrm{H}_{22} \mathrm{O}_{6} \mathrm{~F}_{12} \mathrm{~N}_{2}$ \\
\hline $\mathrm{Fw}$ & 642.72 & 670.78 \\
\hline Temp, ${ }^{\circ} \mathrm{C}$ & $-120 \pm 1^{\circ} \mathrm{C}$ & $-120 \pm 2^{\circ} \mathrm{C}$ \\
\hline$\lambda\left(\right.$ Mo k $\left._{\alpha}\right)$ radiation, $\AA$ & 0.71069 & 0.71069 \\
\hline crystal system & Orthorhombic & monoclinic \\
\hline $\begin{array}{l}\text { space group } \\
\text { unit cell dimens. }(\AA)\end{array}$ & Pbca & $\mathrm{P} 2{ }_{1} / \mathrm{n}$ \\
\hline $\mathrm{a}$ & $15.0844(18)$ & $9.1913(6)$ \\
\hline b & $11.137(2)$ & $17.2805(11)$ \\
\hline $\mathrm{c}$ & $27.709(5)$ & $16.1356(11)$ \\
\hline unit cell angles (deg) & & \\
\hline$\alpha$ & 90.0 & 90.0 \\
\hline$\beta$ & 90.0 & $94.466(1)$ \\
\hline$\gamma$ & 90.0 & 90.0 \\
\hline $\mathrm{V}, \AA^{3}$ & $4654.8(14)$ & $2555.0(3)$ \\
\hline $\mathrm{Z}$ & 8 & 4 \\
\hline$D_{\text {calcd }}, \mathrm{g} / \mathrm{cm}^{3}$ & 1.834 & 1.744 \\
\hline $\mathrm{F}(000)$ & 2528 & 1328 \\
\hline Reflns collected/unique & $\begin{array}{l}39827 / 5806\left(\mathrm{R}_{\mathrm{int}}=\right. \\
0.1225)\end{array}$ & $\begin{array}{l}23439 / 6280\left(\mathrm{R}_{\mathrm{int}}=\right. \\
0.0422)\end{array}$ \\
\hline $\begin{array}{l}\text { Largest diff peak and hole } \\
\left(\mathrm{e}^{-} / \AA^{3}\right)\end{array}$ & 1.423 and -0.870 & 1.715 and -0.971 \\
\hline $\mathrm{R}$ & 0.0593 & 0.063 \\
\hline $\mathrm{R}_{\mathrm{w}}$ & 0.1248 & 0.183 \\
\hline \multicolumn{3}{|c|}{$\begin{array}{l}{ }^{\mathrm{a}} \mathrm{R} 1=\Sigma|| \mathrm{F}_{\mathrm{o}}|-| \mathrm{F}_{\mathrm{c}}|| / \Sigma\left|\mathrm{F}_{\mathrm{o}}\right| \text { and } \mathrm{wR}^{2}=\left\{\Sigma\left[\mathrm{w}\left(\mathrm{F}_{\mathrm{o}}{ }^{2}-\mathrm{F}_{\mathrm{c}}{ }^{2}\right)^{2}\right] / \Sigma\left[\mathrm{w}\left(\mathrm{F}_{\mathrm{o}}{ }^{2}\right)^{2}\right]\right\}^{1 / 2} \text {, where } \\
\mathrm{W}=1 /\left[\sigma^{2}\left(\mathrm{~F}_{\mathrm{o}}^{2}\right)+(0.1551 \mathrm{P})^{2}+0.0000 \mathrm{P}\right] \text { and } \mathrm{P}=\left(\mathrm{F}_{\mathrm{o}}{ }^{2}+2 \mathrm{~F}_{\mathrm{c}}{ }^{2}\right) / 3\end{array}$} \\
\hline
\end{tabular}




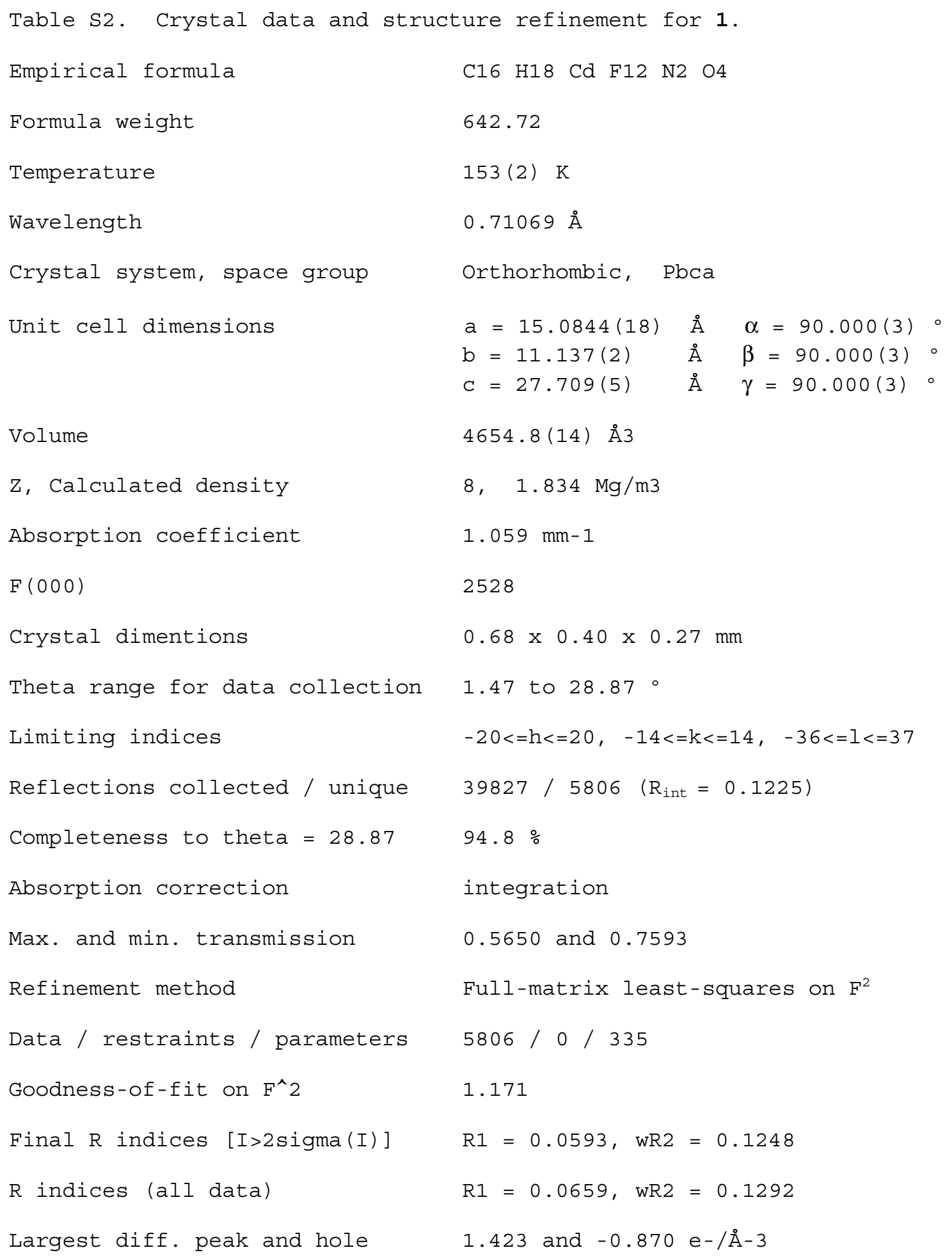


Table S3. Atomic coordinates and equivalent isotropic displacement parameters $\left(A^{\wedge} 2 \times 10^{\wedge} 3\right)$ for 1 .

$\mathrm{U}(\mathrm{eq})$ is defined as one third of the trace of the orthogonalized Uij tensor.

\begin{tabular}{|c|c|c|c|c|}
\hline & $\mathrm{x}$ & $\mathrm{Y}$ & $z$ & $\mathrm{U}(\mathrm{eq})$ \\
\hline $\mathrm{Cd} 1$ & $0.489247(18)$ & $0.05946(3)$ & $0.590929(10)$ & $0.02712(10)$ \\
\hline $\mathrm{F} 1 \mathrm{~A}$ & $0.3702(6)$ & $0.4802(10)$ & $0.6386(4)$ & $0.0499(11)$ \\
\hline F2A & $0.4442(8)$ & $0.3913(7)$ & $0.6949(3)$ & $0.0499(11)$ \\
\hline F3A & $0.5084(7)$ & $0.5279(11)$ & $0.6528(4)$ & $0.0499(11)$ \\
\hline $\mathrm{F} 1 \mathrm{~B}$ & $0.3920(10)$ & $0.5164(14)$ & $0.6258(5)$ & $0.0499(11)$ \\
\hline F2B & $0.3916(10)$ & $0.3873(13)$ & $0.6837(5)$ & $0.0499(11)$ \\
\hline F3B & $0.5075(11)$ & $0.4926(16)$ & $0.6678(6)$ & $0.0499(11)$ \\
\hline $\mathrm{F} 1 \mathrm{C}$ & $0.3648(7)$ & $0.4365(11)$ & $0.6566(5)$ & $0.0499(11)$ \\
\hline $\mathrm{F} 2 \mathrm{C}$ & $0.4859(9)$ & $0.3972(11)$ & $0.6942(4)$ & $0.0499(11)$ \\
\hline $\mathrm{F} 3 \mathrm{C}$ & $0.4827(8)$ & $0.5421(12)$ & $0.6439(5)$ & $0.0499(11)$ \\
\hline F4 & $0.5749(3)$ & $0.4142(6)$ & $0.47176(18)$ & $0.116(2)$ \\
\hline F5 & $0.6827(3)$ & $0.4359(3)$ & $0.52132(14)$ & $0.0762(12)$ \\
\hline F6 & $0.6734(2)$ & $0.2827(3)$ & $0.47827(12)$ & $0.0563(8)$ \\
\hline F7 & $0.6852(3)$ & $0.0208(6)$ & $0.73062(13)$ & $0.1033(17)$ \\
\hline F8 & $0.7509(4)$ & $0.1499(4)$ & 0.69085 (19) & $0.117(2)$ \\
\hline F9 & $0.8007(3)$ & $-0.0267(5)$ & $0.69408(18)$ & $0.1046(18)$ \\
\hline F10 & $0.6511(2)$ & $-0.2723(3)$ & $0.51628(12)$ & $0.0566(8)$ \\
\hline F11 & $0.6081(3)$ & $-0.3467(3)$ & $0.58372(17)$ & $0.0784(12)$ \\
\hline $\mathrm{F} 12$ & $0.7446(2)$ & $-0.3030(3)$ & $0.57288(12)$ & $0.0541(8)$ \\
\hline 01 & $0.4367(2)$ & $0.2393(3)$ & $0.61744(11)$ & $0.0360(7)$ \\
\hline $\mathrm{O} 2$ & $0.55106(19)$ & $0.1881(3)$ & $0.53423(10)$ & $0.0297(6)$ \\
\hline 03 & $0.6095(2)$ & $0.0798(3)$ & $0.63963(11)$ & $0.0356(7)$ \\
\hline 04 & $0.57601(19)$ & $-0.0941(3)$ & $0.56275(10)$ & $0.0329(6)$ \\
\hline N1 & $0.3994(2)$ & $-0.0514(3)$ & $0.64618(11)$ & $0.0289(7)$ \\
\hline N2 & $0.3771(2)$ & $-0.0010(4)$ & $0.54160(13)$ & $0.0316(7)$ \\
\hline $\mathrm{H} 2 \mathrm{C}$ & $0.390(4)$ & $-0.062(5)$ & $0.524(2)$ & $0.045(15)$ \\
\hline $\mathrm{H} 2 \mathrm{D}$ & $0.365(3)$ & $0.062(5)$ & $0.5226(19)$ & $0.037(13)$ \\
\hline $\mathrm{C} 1$ & $0.3344(3)$ & $-0.1127(4)$ & $0.61461(15)$ & $0.0321(8)$ \\
\hline $\mathrm{H} 1 \mathrm{~A}$ & 0.2828 & -0.1378 & 0.6343 & 0.039 \\
\hline $\mathrm{H} 1 \mathrm{~B}$ & 0.3620 & -0.1859 & 0.6011 & 0.039 \\
\hline $\mathrm{C} 2$ & $0.3022(3)$ & $-0.0334(4)$ & $0.57332(16)$ & $0.0337(9)$ \\
\hline $\mathrm{H} 2 \mathrm{~A}$ & 0.2566 & -0.0768 & 0.5544 & 0.040 \\
\hline $\mathrm{H} 2 \mathrm{~B}$ & 0.2749 & 0.0404 & 0.5865 & 0.040 \\
\hline C3 & $0.4545(3)$ & $-0.1427(4)$ & $0.67178(16)$ & $0.0375(9)$ \\
\hline $\mathrm{H} 3 \mathrm{~A}$ & 0.5014 & -0.1003 & 0.6900 & 0.045 \\
\hline $\mathrm{H} 3 \mathrm{~B}$ & 0.4841 & -0.1935 & 0.6473 & 0.045 \\
\hline $\mathrm{C} 4$ & $0.4044(4)$ & $-0.2247(5)$ & $0.70667(18)$ & $0.0472(12)$ \\
\hline $\mathrm{H} 4 \mathrm{~A}$ & 0.3796 & -0.1766 & 0.7330 & 0.071 \\
\hline $\mathrm{H} 4 \mathrm{~B}$ & 0.4453 & -0.2846 & 0.7199 & 0.071 \\
\hline $\mathrm{H} 4 \mathrm{C}$ & 0.3563 & -0.2654 & 0.6894 & 0.071 \\
\hline C5 & $0.3531(3)$ & $0.0310(4)$ & $0.68014(15)$ & $0.0359(9)$ \\
\hline $\mathrm{H} 5 \mathrm{~A}$ & 0.3080 & -0.0152 & 0.6982 & 0.043 \\
\hline $\mathrm{H} 5 \mathrm{~B}$ & 0.3218 & 0.0934 & 0.6613 & 0.043 \\
\hline $\mathrm{C} 6$ & $0.4146(4)$ & $0.0917(5)$ & $0.71577(16)$ & $0.0452(12)$ \\
\hline $\mathrm{H} 6 \mathrm{~A}$ & 0.4329 & 0.0337 & 0.7404 & 0.068 \\
\hline $\mathrm{H} 6 \mathrm{~B}$ & 0.3836 & 0.1589 & 0.7312 & 0.068 \\
\hline
\end{tabular}




$\begin{array}{lllll}\text { H6C } & 0.4670 & 0.1218 & 0.6988 & 0.068 \\ \text { C7 } & 0.4763(3) & 0.3375(4) & 0.61311(15) & 0.0321(8) \\ \text { C8 } & 0.5390(3) & 0.3715(4) & 0.57903(16) & 0.0342(9) \\ \text { H8 } & 0.5641 & 0.4495 & 0.5814 & 0.041 \\ \text { C9 } & 0.5670(3) & 0.2966(4) & 0.54139(14) & 0.0282(8) \\ \text { C10 } & 0.4490(3) & 0.4320(5) & 0.65012(17) & 0.0405(10) \\ \text { C11 } & 0.6236(3) & 0.3588(4) & 0.50256(17) & 0.0386(10) \\ \text { C12 } & 0.6703(3) & 0.0055(4) & 0.64658(15) & 0.0340(9) \\ \text { C13 } & 0.6901(3) & -0.1004(4) & 0.62229(16) & 0.0348(9) \\ \text { H13 } & 0.7396 & -0.1463 & 0.6327 & 0.042 \\ \text { C14 } & 0.6405(3) & -0.1419(4) & 0.58350(15) & 0.0300(8) \\ \text { C15 } & 0.7286(3) & 0.0380(5) & 0.69034(19) & 0.0475(12) \\ \text { C16 } & 0.6622(3) & -0.2668(4) & 0.56387(19) & 0.0412(10)\end{array}$


Table S4. Bond lengths [A] and angles [deg] for 1.

\begin{tabular}{|c|c|c|}
\hline $\mathrm{Cd} 1$ & $\mathrm{O} 3$ & $2.272(3)$ \\
\hline $\mathrm{Cd1}$ & $\mathrm{O} 1$ & $2.276(3)$ \\
\hline $\mathrm{Cd} 1$ & N2 & $2.277(4)$ \\
\hline $\mathrm{Cd} 1$ & O4 & $2.290(3)$ \\
\hline $\mathrm{Cd} 1$ & $\mathrm{O} 2$ & $2.322(3)$ \\
\hline $\mathrm{Cd1}$ & $\mathrm{N} 1$ & $2.388(3)$ \\
\hline $\mathrm{F} 1 \mathrm{~A}$ & $F 1 B$ & $0.628(15)$ \\
\hline $\mathrm{F} 1 \mathrm{~A}$ & $\mathrm{~F} 1 \mathrm{C}$ & $0.702(12)$ \\
\hline $\mathrm{F} 1 \mathrm{~A}$ & $\mathrm{C} 10$ & $1.343(10)$ \\
\hline $\mathrm{F} 1 \mathrm{~A}$ & $F 2 B$ & $1.654(19)$ \\
\hline $\mathrm{F} 2 \mathrm{~A}$ & $\mathrm{~F} 2 \mathrm{C}$ & $0.632(11)$ \\
\hline $\mathrm{F} 2 \mathrm{~A}$ & F2B & $0.854(15)$ \\
\hline $\mathrm{F} 2 \mathrm{~A}$ & $\mathrm{C} 10$ & $1.324(9)$ \\
\hline $\mathrm{F} 2 \mathrm{~A}$ & F3B & $1.66(2)$ \\
\hline $\mathrm{F} 2 \mathrm{~A}$ & F1C & $1.678(16)$ \\
\hline F3A & F3B & $0.573(18)$ \\
\hline F3A & $\mathrm{C} 10$ & $1.396(13)$ \\
\hline$F 1 B$ & F1C & $1.30(2)$ \\
\hline $\mathrm{F} 1 \mathrm{~B}$ & $\mathrm{C} 10$ & $1.440(16)$ \\
\hline $\mathrm{F} 1 \mathrm{~B}$ & F3C & $1.48(2)$ \\
\hline F2B & F1C & $1.013(18)$ \\
\hline $\mathrm{F} 2 \mathrm{~B}$ & $\mathrm{C} 10$ & $1.365(14)$ \\
\hline F2B & F2C & $1.456(18)$ \\
\hline F3B & F3C & $0.94(2)$ \\
\hline F3B & $\mathrm{C} 10$ & $1.214(18)$ \\
\hline F3B & F2C & $1.33(2)$ \\
\hline $\mathrm{F} 1 \mathrm{C}$ & $\mathrm{C} 10$ & $1.283(12)$ \\
\hline $\mathrm{F} 2 \mathrm{C}$ & $\mathrm{C} 10$ & $1.397(13)$ \\
\hline F3C & $\mathrm{C} 10$ & $1.339(15)$ \\
\hline F4 & $\mathrm{C} 11$ & $1.284(6)$ \\
\hline F5 & $\mathrm{C} 11$ & $1.343(6)$ \\
\hline F6 & $\mathrm{C} 11$ & $1.317(5)$ \\
\hline F7 & C15 & $1.308(7)$ \\
\hline F8 & C15 & $1.291(7)$ \\
\hline F9 & C15 & $1.309(7)$ \\
\hline F10 & $\mathrm{C} 16$ & $1.331(6)$ \\
\hline F11 & $\mathrm{C} 16$ & $1.327(6)$ \\
\hline F12 & $\mathrm{C} 16$ & $1.329(5)$ \\
\hline $\mathrm{O} 1$ & $\mathrm{C} 7$ & $1.252(5)$ \\
\hline $\mathrm{O} 2$ & $\mathrm{C} 9$ & $1.248(5)$ \\
\hline 03 & $\mathrm{C} 12$ & $1.250(5)$ \\
\hline 04 & $\mathrm{C} 14$ & $1.250(5)$ \\
\hline $\mathrm{N1}$ & $\mathrm{C} 1$ & $1.480(5)$ \\
\hline $\mathrm{N1}$ & $\mathrm{C} 5$ & $1.487(5)$ \\
\hline $\mathrm{N1}$ & C3 & $1.493(5)$ \\
\hline $\mathrm{N} 2$ & $\mathrm{C} 2$ & $1.476(5)$ \\
\hline $\mathrm{N} 2$ & $\mathrm{H} 2 \mathrm{C}$ & $0.87(6)$ \\
\hline $\mathrm{N} 2$ & $\mathrm{H} 2 \mathrm{D}$ & $0.90(5)$ \\
\hline $\mathrm{C} 1$ & $\mathrm{C} 2$ & $1.524(6)$ \\
\hline $\mathrm{C} 1$ & $\mathrm{H} 1 \mathrm{~A}$ & 0.9900 \\
\hline $\mathrm{C} 1$ & $\mathrm{H} 1 \mathrm{~B}$ & 0.9900 \\
\hline $\mathrm{C} 2$ & $\mathrm{H} 2 \mathrm{~A}$ & 0.9900 \\
\hline $\mathrm{C} 2$ & $\mathrm{H} 2 \mathrm{~B}$ & 0.9900 \\
\hline
\end{tabular}




\begin{tabular}{|c|c|c|}
\hline C3 & C4 & $1.529(6)$ \\
\hline $\mathrm{C} 3$ & $\mathrm{H} 3 \mathrm{~A}$ & 0.9900 \\
\hline $\mathrm{C} 3$ & Н3В & 0.9900 \\
\hline $\mathrm{C} 4$ & $\mathrm{H} 4 \mathrm{~A}$ & 0.9800 \\
\hline $\mathrm{C} 4$ & $\mathrm{H} 4 \mathrm{~B}$ & 0.9800 \\
\hline $\mathrm{C} 4$ & $\mathrm{H} 4 \mathrm{C}$ & 0.9800 \\
\hline $\mathrm{C} 5$ & $\mathrm{C} 6$ & $1.514(7)$ \\
\hline $\mathrm{C} 5$ & H5A & 0.9900 \\
\hline $\mathrm{C} 5$ & H5B & 0.9900 \\
\hline $\mathrm{C} 6$ & $\mathrm{H} 6 \mathrm{~A}$ & 0.9800 \\
\hline $\mathrm{C} 6$ & H6B & 0.9800 \\
\hline $\mathrm{C} 6$ & $\mathrm{H} 6 \mathrm{C}$ & 0.9800 \\
\hline $\mathrm{C} 7$ & C8 & $1.389(6)$ \\
\hline $\mathrm{C} 7$ & $\mathrm{C} 10$ & $1.526(6)$ \\
\hline C8 & C9 & $1.401(6)$ \\
\hline $\mathrm{C} 8$ & H8 & 0.9500 \\
\hline C9 & $\mathrm{C} 11$ & $1.539(6)$ \\
\hline $\mathrm{C} 12$ & $\mathrm{C} 13$ & $1.390(7)$ \\
\hline $\mathrm{C} 12$ & C15 & $1.540(6)$ \\
\hline C13 & $\mathrm{C} 14$ & $1.389(6)$ \\
\hline C13 & $\mathrm{H} 13$ & 0.9500 \\
\hline C14 & $\mathrm{C} 16$ & $1.529(6)$ \\
\hline 03 & Ca1 O1 & $89.93(11)$ \\
\hline 03 & Ca1 N2 & $168.30(13)$ \\
\hline 01 & Ca1 N2 & $101.25(13)$ \\
\hline 03 & Ca1 O4 & $79.66(11)$ \\
\hline 01 & Cal O4 & $165.02(11)$ \\
\hline N2 & Ca1 O4 & $89.96(13)$ \\
\hline 03 & $\mathrm{Cd} 1 \mathrm{O} 2$ & $91.13(11)$ \\
\hline 01 & $\mathrm{Ca} 1 \mathrm{O} 2$ & $79.34(10)$ \\
\hline N2 & Ca1 O2 & $94.28(12)$ \\
\hline 04 & $\mathrm{Ca1} \mathrm{O} 2$ & $90.03(11)$ \\
\hline 03 & Cal N1 & $97.12(12)$ \\
\hline 01 & Cal N1 & $92.87(11)$ \\
\hline N2 & Cal N1 & $79.07(12)$ \\
\hline 04 & Cd1 N1 & $99.04(12)$ \\
\hline $\mathrm{O} 2$ & Cd1 N1 & $168.68(11)$ \\
\hline$F 1 B$ & $\mathrm{~F} 1 \mathrm{~A} \quad \mathrm{~F} 1 \mathrm{C}$ & $155(3)$ \\
\hline$F 1 B$ & $\mathrm{~F} 1 \mathrm{~A} \quad \mathrm{C} 10$ & $85.8(18)$ \\
\hline $\mathrm{F} 1 \mathrm{C}$ & $\mathrm{F} 1 \mathrm{~A} \quad \mathrm{C} 10$ & $69.8(13)$ \\
\hline$F 1 B$ & $\mathrm{~F} 1 \mathrm{~A} \quad \mathrm{~F} 2 \mathrm{~B}$ & $136(2)$ \\
\hline $\mathrm{F} 1 \mathrm{C}$ & $\mathrm{F} 1 \mathrm{~A} \quad \mathrm{~F} 2 \mathrm{~B}$ & $18.4(13)$ \\
\hline C10 & $\mathrm{F} 1 \mathrm{~A} \quad \mathrm{~F} 2 \mathrm{~B}$ & $53.0(6)$ \\
\hline $\mathrm{F} 2 \mathrm{C}$ & F2A F $2 B$ & $157(2)$ \\
\hline $\mathrm{F} 2 \mathrm{C}$ & F2A C10 & $83.1(15)$ \\
\hline$F 2 B$ & F2A C10 & $74.1(12)$ \\
\hline $\mathrm{F} 2 \mathrm{C}$ & F2A F3B & $48.8(15)$ \\
\hline F2B & F2A F3B & $113.9(15)$ \\
\hline C10 & F2A F3B & $46.4(7)$ \\
\hline $\mathrm{F} 2 \mathrm{C}$ & F2A F1C & $131.1(18)$ \\
\hline F2B & $\mathrm{F} 2 \mathrm{~A} \quad \mathrm{~F} 1 \mathrm{C}$ & $28.5(11)$ \\
\hline C10 & $\mathrm{F} 2 \mathrm{~A} \quad \mathrm{~F} 1 \mathrm{C}$ & $48.9(5)$ \\
\hline F3B & F2A F1C & $85.4(9)$ \\
\hline F3B & F3A C10 & $60(2)$ \\
\hline $\mathrm{F} 1 \mathrm{~A}$ & F1B F1C & $13.4(15)$ \\
\hline $\mathrm{F} 1 \mathrm{~A}$ & F1B C10 & $68.4(18)$ \\
\hline
\end{tabular}




\begin{tabular}{|c|c|c|c|}
\hline F1C & $\mathrm{F} 1 \mathrm{~B}$ & $\mathrm{C} 10$ & $55.6(8)$ \\
\hline $\mathrm{F} 1 \mathrm{~A}$ & $\mathrm{~F} 1 \mathrm{~B}$ & $\mathrm{~F} 3 \mathrm{C}$ & $115(2)$ \\
\hline $\mathrm{F} 1 \mathrm{C}$ & $\mathrm{F} 1 \mathrm{~B}$ & $\mathrm{~F} 3 \mathrm{C}$ & $101.6(12)$ \\
\hline $\mathrm{C} 10$ & $\mathrm{~F} 1 \mathrm{~B}$ & $\mathrm{~F} 3 \mathrm{C}$ & $54.4(7)$ \\
\hline $\mathrm{F} 2 \mathrm{~A}$ & F2B & $\mathrm{F} 1 \mathrm{C}$ & $127.8(19)$ \\
\hline $\mathrm{F} 2 \mathrm{~A}$ & $\mathrm{~F} 2 \mathrm{~B}$ & $\mathrm{C} 10$ & $68.9(11)$ \\
\hline $\mathrm{F} 1 \mathrm{C}$ & $\mathrm{F} 2 \mathrm{~B}$ & $\mathrm{C} 10$ & $63.3(10)$ \\
\hline $\mathrm{F} 2 \mathrm{~A}$ & $\mathrm{~F} 2 \mathrm{~B}$ & $\mathrm{~F} 2 \mathrm{C}$ & $10.0(9)$ \\
\hline $\mathrm{F} 1 \mathrm{C}$ & $\mathrm{F} 2 \mathrm{~B}$ & $\mathrm{~F} 2 \mathrm{C}$ & $119.7(14)$ \\
\hline $\mathrm{C} 10$ & $\mathrm{~F} 2 \mathrm{~B}$ & $\mathrm{~F} 2 \mathrm{C}$ & $59.2(8)$ \\
\hline $\mathrm{F} 2 \mathrm{~A}$ & $\mathrm{~F} 2 \mathrm{~B}$ & $F 1 A$ & $115.1(15)$ \\
\hline $\mathrm{F} 1 \mathrm{C}$ & $\mathrm{F} 2 \mathrm{~B}$ & $F 1 A$ & $12.7(8)$ \\
\hline $\mathrm{C} 10$ & $\mathrm{~F} 2 \mathrm{~B}$ & $F 1 A$ & $51.7(6)$ \\
\hline $\mathrm{F} 2 \mathrm{C}$ & $\mathrm{F} 2 \mathrm{~B}$ & $F 1 A$ & $107.1(11)$ \\
\hline F3A & F3B & $\mathrm{F} 3 \mathrm{C}$ & $25(2)$ \\
\hline F3A & F3B & $\mathrm{C} 10$ & $96(3)$ \\
\hline $\mathrm{F} 3 \mathrm{C}$ & F3B & $\mathrm{C} 10$ & $75.7(13)$ \\
\hline F3A & F3B & $\mathrm{F} 2 \mathrm{C}$ & $163(3)$ \\
\hline $\mathrm{F} 3 \mathrm{C}$ & F3B & $\mathrm{F} 2 \mathrm{C}$ & $139.4(19)$ \\
\hline C10 & F3B & F2C & $66.4(11)$ \\
\hline F3A & F3B & $\mathrm{F} 2 \mathrm{~A}$ & $144(3)$ \\
\hline $\mathrm{F} 3 \mathrm{C}$ & F3B & $\mathrm{F} 2 \mathrm{~A}$ & $119.3(16)$ \\
\hline C10 & F3B & $\mathrm{F} 2 \mathrm{~A}$ & $52.1(8)$ \\
\hline $\mathrm{F} 2 \mathrm{C}$ & F3B & $\mathrm{F} 2 \mathrm{~A}$ & $21.0(6)$ \\
\hline $\mathrm{F} 1 \mathrm{~A}$ & $\mathrm{~F} 1 \mathrm{C}$ & F2B & $149(2)$ \\
\hline $\mathrm{F} 1 \mathrm{~A}$ & $\mathrm{~F} 1 \mathrm{C}$ & $\mathrm{C} 10$ & $79.3(13)$ \\
\hline F2B & $\mathrm{F} 1 \mathrm{C}$ & $\mathrm{C} 10$ & $71.9(11)$ \\
\hline $\mathrm{F} 1 \mathrm{~A}$ & $\mathrm{~F} 1 \mathrm{C}$ & $F 1 B$ & $11.9(13)$ \\
\hline F2B & $\mathrm{F} 1 \mathrm{C}$ & $F 1 B$ & $137.0(15)$ \\
\hline C10 & $\mathrm{F} 1 \mathrm{C}$ & $F 1 B$ & $67.8(9)$ \\
\hline $\mathrm{F} 1 \mathrm{~A}$ & $\mathrm{~F} 1 \mathrm{C}$ & $\mathrm{F} 2 \mathrm{~A}$ & $125.2(16)$ \\
\hline F2B & $\mathrm{F} 1 \mathrm{C}$ & $F 2 A$ & $23.7(9)$ \\
\hline C10 & $\mathrm{F} 1 \mathrm{C}$ & F $2 A$ & $51.0(5)$ \\
\hline$F 1 B$ & $\mathrm{~F} 1 \mathrm{C}$ & $\mathrm{F} 2 \mathrm{~A}$ & $113.4(10)$ \\
\hline $\mathrm{F} 2 \mathrm{~A}$ & $\mathrm{~F} 2 \mathrm{C}$ & F3B & $110.2(18)$ \\
\hline $\mathrm{F} 2 \mathrm{~A}$ & $\mathrm{~F} 2 \mathrm{C}$ & $\mathrm{C} 10$ & $70.2(15)$ \\
\hline F3B & $\mathrm{F} 2 \mathrm{C}$ & $\mathrm{C} 10$ & $52.8(8)$ \\
\hline $\mathrm{F} 2 \mathrm{~A}$ & $\mathrm{~F} 2 \mathrm{C}$ & F2B & $13.5(12)$ \\
\hline F3B & $\mathrm{F} 2 \mathrm{C}$ & $F 2 B$ & $100.9(11)$ \\
\hline $\mathrm{C} 10$ & $\mathrm{~F} 2 \mathrm{C}$ & $F 2 B$ & $57.1(8)$ \\
\hline F3B & $\mathrm{F} 3 \mathrm{C}$ & $\mathrm{C} 10$ & $61.5(13)$ \\
\hline F3B & F3C & $F 1 B$ & $119.4(17)$ \\
\hline $\mathrm{C} 10$ & F3C & $F 1 B$ & $61.1(8)$ \\
\hline $\mathrm{C} 7$ & 01 & Cal & $124.9(3)$ \\
\hline C9 & $\mathrm{O} 2$ & $\mathrm{Cd} 1$ & $124.6(3)$ \\
\hline $\mathrm{C} 12$ & $\mathrm{O} 3$ & $\mathrm{Cd} 1$ & $127.7(3)$ \\
\hline C14 & O4 & $\mathrm{Cd} 1$ & $127.3(3)$ \\
\hline $\mathrm{C} 1$ & N1 & C5 & $110.4(3)$ \\
\hline $\mathrm{C} 1$ & N1 & C3 & $109.6(3)$ \\
\hline C5 & $\mathrm{N} 1$ & C3 & $112.4(3)$ \\
\hline $\mathrm{C} 1$ & N1 & $\mathrm{Cd} 1$ & $103.6(2)$ \\
\hline C5 & N1 & $\mathrm{Cd} 1$ & $110.7(3)$ \\
\hline C3 & N1 & $\mathrm{CdI}$ & $109.9(2)$ \\
\hline $\mathrm{C} 2$ & N2 & $\mathrm{Cd} 1$ & $106.5(3)$ \\
\hline $\mathrm{C} 2$ & N2 & $\mathrm{H} 2 \mathrm{C}$ & $109(4)$ \\
\hline Cal & N2 & $\mathrm{H} 2 \mathrm{C}$ & $114(4)$ \\
\hline $\mathrm{C} 2$ & N2 & $\mathrm{H} 2 \mathrm{D}$ & $113(3)$ \\
\hline
\end{tabular}




\begin{tabular}{|c|c|c|}
\hline $2 \mathrm{C}$ & N2 & $12 \mathrm{D}$ \\
\hline & $\mathrm{C} 1$ & \\
\hline & $\mathrm{C} 1$ & 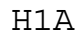 \\
\hline & C1 & $11 \mathrm{~A}$ \\
\hline & $\mathrm{C} 1$ & $\mathrm{H} 1 \mathrm{~B}$ \\
\hline & $\mathrm{C} 1$ & $\mathrm{H} 1 \mathrm{~B}$ \\
\hline & C1 & $\mathrm{H} 1 \mathrm{~B}$ \\
\hline & $\mathrm{C} 2$ & \\
\hline & $\mathrm{C} 2$ & $\mathrm{H} 2 \mathrm{~A}$ \\
\hline & $\mathrm{C} 2$ & $\mathrm{H} 2 \mathrm{~A}$ \\
\hline & $\mathrm{C} 2$ & $12 \mathrm{~B}$ \\
\hline & $\mathrm{C} 2$ & $12 B$ \\
\hline $2 \mathrm{~A}$ & $\mathrm{C} 2$ & $12 \mathrm{~B}$ \\
\hline & C3 & $\mathrm{C} 4$ \\
\hline & $\mathrm{C} 3$ & $\mathrm{H} 3 \mathrm{~A}$ \\
\hline & C3 & $13 \mathrm{~A}$ \\
\hline & $\mathrm{C} 3$ & $13 \mathrm{~B}$ \\
\hline & C3 & {$[3 \mathrm{E}$} \\
\hline 3. & C3 & $13 \mathrm{~B}$ \\
\hline & $\mathrm{C} 4$ & $14 \mathrm{~A}$ \\
\hline & $\mathrm{C} 4$ & $14 \mathrm{~B}$ \\
\hline $\pm A$ & $\mathrm{C} 4$ & $4 \mathrm{~B}$ \\
\hline & $\mathrm{C} 4$ & $4 \mathrm{C}$ \\
\hline 4 & $\mathrm{C} 4$ & $4 \mathrm{C}$ \\
\hline . & $\mathrm{C} 4$ & $14 \mathrm{C}$ \\
\hline & C5 & C6 \\
\hline & C5 & $45 \mathrm{~A}$ \\
\hline & $\mathrm{C} 5$ & $15 \mathrm{~A}$ \\
\hline & C5 & $15 B$ \\
\hline 6 & $\mathrm{C} 5$ & $15 B$ \\
\hline $5 \mathrm{~A}$ & C5 & $45 B$ \\
\hline 5 & C6 & $16 \mathrm{~A}$ \\
\hline 5 & C6 & $\mathrm{H} 6 \mathrm{~B}$ \\
\hline $6 \mathrm{~A}$ & C6 & $16 \mathrm{~B}$ \\
\hline 5 & C6 & $16 \mathrm{C}$ \\
\hline $6 \mathrm{~A}$ & C6 & $16 \mathrm{C}$ \\
\hline $6 \mathrm{~B}$ & C6 & $\mathrm{H} 6 \mathrm{C}$ \\
\hline 1 & C7 & C8 \\
\hline 1 & C7 & $\mathrm{C} 10$ \\
\hline 8 & C7 & $\mathrm{C} 1 \mathrm{C}$ \\
\hline C7 & C8 & C9 \\
\hline 7 & C8 & H8 \\
\hline C9 & C8 & H8 \\
\hline 2 & C9 & C8 \\
\hline 2 & C9 & $\mathrm{C} 11$ \\
\hline 8 & C9 & $\mathrm{C} 11$ \\
\hline $3 B$ & $\mathrm{C} 10$ & $\mathrm{~F} 1 \mathrm{C}$ \\
\hline $3 B$ & $\mathrm{C} 10$ & $\mathrm{~F} 2 \mathrm{~F}$ \\
\hline $1 \mathrm{C}$ & $\mathrm{C} 10$ & $\mathrm{~F} 2 \mathrm{~A}$ \\
\hline $3 B$ & $\mathrm{C} 10$ & $\mathrm{~F} 3 \mathrm{C}$ \\
\hline $\mathrm{F} 1 \mathrm{C}$ & $\mathrm{C} 10$ & $\mathrm{~F} 3 \mathrm{C}$ \\
\hline $\mathrm{F} 2 \mathrm{~A}$ & $\mathrm{C} 10$ & $\mathrm{~F} 3 \mathrm{C}$ \\
\hline F3B & $\mathrm{C} 10$ & $F \perp A$ \\
\hline & $\mathrm{C} 10$ & $\mathrm{~F} 17$ \\
\hline $2 \mathrm{~A}$ & $\mathrm{C} 10$ & $\mathrm{~F} 1 \mathrm{I}$ \\
\hline & $\mathrm{C} 10$ & \\
\hline
\end{tabular}

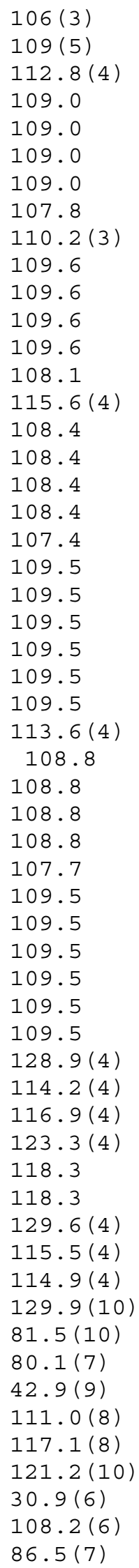




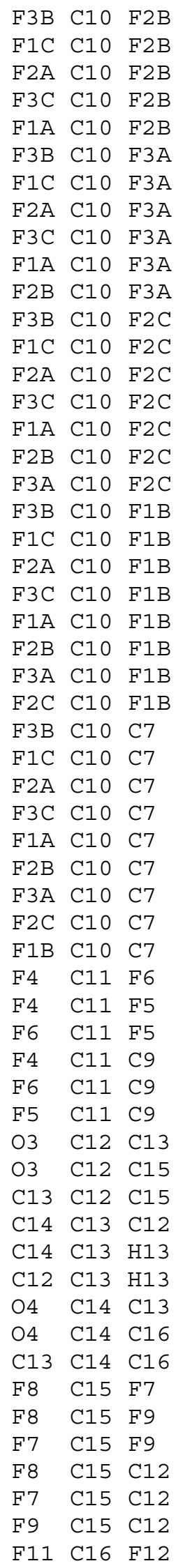

$112.9(11)$

$44.8(8)$

$37.0(7)$

$131.6(9)$

$75.3(8)$

$24.1(8)$

$126.7(7)$

$104.3(7)$

$20.3(6)$

$106.0(6)$

$130.6(8)$

$60.8(10)$

$106.4(8)$

$26.7(5)$

$102.5(8)$

$132.2(7)$

$63.6(8)$

$84.9(7)$

$105.1(11)$

$56.6(8)$

$129.0(8)$

$64.5(9)$

$25.8(6)$

$100.2(9)$

$84.8(8)$

$145.9(8)$

$117.2(8)$

$112.9(6)$

$114.1(5)$

$116.2(6)$

$110.8(5)$

$112.2(7)$

$112.9(5)$

$106.7(6)$

$107.3(6)$

$107.2(5)$

$109.3(5)$

$103.3(4)$

$111.3(4)$

$112.6(4)$

$112.7(4)$

$130.1(4)$

$112.6(4)$

$117.2(4)$

$122.8(4)$

118.6

118.6

$129.3(4)$

$113.0(4)$

$117.6(4)$

$105.2(6)$

$108.3(5)$

$105.6(5)$

$112.6(4)$

$110.6(4)$

$114.0(5)$

$107.1(4)$ 
F11 C16 F10

F12 C16 F10

F11 C16 C14

F12 C16 C14

F10 C16 C14
$107.6(4)$

$106.9(4)$

$109.3(4)$

$114.1(4)$

$111.6(4)$ 
Table S5. Anisotropic displacement parameters (A^2) for 1.

The anisotropic displacement factor exponent takes the form: $-2 \mathrm{pi}^{\wedge} 2$ [ h^2 $\mathrm{a}^{{ }^{\wedge} 2} \mathrm{U11}+\ldots+2 \mathrm{hk} \mathrm{a}^{*} \mathrm{~b} * \mathrm{U} 12$ ]

\begin{tabular}{|c|c|c|c|c|c|c|}
\hline & $\mathrm{U} 11$ & $\mathrm{U} 22$ & U33 & U23 & U13 & $\mathrm{U} 12$ \\
\hline $\mathrm{Cd} 1$ & $0.03037(16)$ & $0.02735(16)$ & $0.02363(15)$ & $0.00273(11)$ & $0.00217(10)$ & $-0.00064(11)$ \\
\hline$F 1 A$ & $0.050(2)$ & $0.050(2)$ & $0.049(2)$ & $-0.0117(15)$ & $0.009(2)$ & $0.004(2)$ \\
\hline $\mathrm{F} 2 \mathrm{~A}$ & $0.050(2)$ & $0.050(2)$ & $0.049(2)$ & $-0.0117(15)$ & $0.009(2)$ & $0.004(2)$ \\
\hline F $3 A$ & $0.050(2)$ & $0.050(2)$ & $0.049(2)$ & $-0.0117(15)$ & $0.009(2)$ & $0.004(2)$ \\
\hline $\mathrm{F} 1 \mathrm{~B}$ & $0.050(2)$ & $0.050(2)$ & $0.049(2)$ & $-0.0117(15)$ & $0.009(2)$ & $0.004(2)$ \\
\hline $\mathrm{F} 2 \mathrm{~B}$ & $0.050(2)$ & $0.050(2)$ & $0.049(2)$ & $-0.0117(15)$ & $0.009(2)$ & $0.004(2)$ \\
\hline F $3 B$ & $0.050(2)$ & $0.050(2)$ & $0.049(2)$ & $-0.0117(15)$ & $0.009(2)$ & $0.004(2)$ \\
\hline $\mathrm{F} 1 \mathrm{C}$ & $0.050(2)$ & $0.050(2)$ & $0.049(2)$ & $-0.0117(15)$ & $0.009(2)$ & $0.004(2)$ \\
\hline $\mathrm{F} 2 \mathrm{C}$ & $0.050(2)$ & $0.050(2)$ & $0.049(2)$ & $-0.0117(15)$ & $0.009(2)$ & $0.004(2)$ \\
\hline $\mathrm{F} 3 \mathrm{C}$ & $0.050(2)$ & $0.050(2)$ & $0.049(2)$ & $-0.0117(15)$ & $0.009(2)$ & $0.004(2)$ \\
\hline F4 & $0.065(2)$ & $0.187(5)$ & $0.098(3)$ & $0.111(4)$ & $0.023(2)$ & $0.033(3)$ \\
\hline F5 & $0.094(3)$ & $0.067(2)$ & $0.068(2)$ & $-0.0226(19)$ & $0.036(2)$ & $-0.046(2)$ \\
\hline F6 & $0.0672(19)$ & $0.0491(18)$ & $0.0525(18)$ & $-0.0053(14)$ & $0.0304(15)$ & $-0.0082(15)$ \\
\hline F7 & $0.092(3)$ & $0.184(5)$ & $0.0340(18)$ & $0.001(2)$ & $-0.0152(19)$ & $-0.021(3)$ \\
\hline F8 & $0.167(5)$ & $0.074(3)$ & $0.109(4)$ & $0.010(3)$ & $-0.092(4)$ & $-0.048(3)$ \\
\hline F9 & $0.069(2)$ & $0.142(4)$ & $0.103(3)$ & $-0.054(3)$ & $-0.054(2)$ & $0.035(3)$ \\
\hline F10 & $0.0601(18)$ & $0.056(2)$ & $0.0538(18)$ & $-0.0239(15)$ & $-0.0098(15)$ & $0.0186(15)$ \\
\hline F11 & $0.083(3)$ & $0.0335(17)$ & $0.118(3)$ & $-0.0067(19)$ & $0.039(2)$ & $-0.0166(17)$ \\
\hline $\mathrm{F} 12$ & $0.0528(17)$ & $0.0497(18)$ & $0.0597(18)$ & $0.0005(15)$ & $-0.0049(14)$ & $0.0237(15)$ \\
\hline $\mathrm{O} 1$ & $0.0408(16)$ & $0.0312(16)$ & $0.0359(15)$ & $-0.0001(12)$ & $0.0104(12)$ & $-0.0003(13)$ \\
\hline $\mathrm{O} 2$ & $0.0351(14)$ & $0.0264(14)$ & $0.0276(13)$ & $0.0005(11)$ & $0.0023(11)$ & $-0.0030(12)$ \\
\hline $\mathrm{O} 3$ & $0.0368(15)$ & $0.0351(17)$ & $0.0347(15)$ & $-0.0033(12)$ & $-0.0068(12)$ & $-0.0022(13)$ \\
\hline 04 & $0.0337(14)$ & $0.0366(16)$ & $0.0286(14)$ & $-0.0029(12)$ & $-0.0036(11)$ & $0.0057(12)$ \\
\hline N1 & $0.0352(17)$ & $0.0305(17)$ & $0.0210(14)$ & $0.0030(13)$ & $0.0023(12)$ & $-0.0004(14)$ \\
\hline N2 & $0.0366(18)$ & $0.033(2)$ & $0.0250(16)$ & $0.0024(15)$ & $-0.0006(13)$ & $0.0000(16)$ \\
\hline $\mathrm{C} 1$ & $0.035(2)$ & $0.034(2)$ & $0.0277(19)$ & $0.0011(16)$ & $0.0028(15)$ & $-0.0036(17)$ \\
\hline $\mathrm{C} 2$ & $0.0299(19)$ & $0.039(2)$ & $0.032(2)$ & $0.0004(17)$ & $-0.0011(16)$ & $0.0015(17)$ \\
\hline $\mathrm{C} 3$ & $0.040(2)$ & $0.040(2)$ & $0.032(2)$ & $0.0095(18)$ & $0.0006(17)$ & $0.0039(19)$ \\
\hline $\mathrm{C} 4$ & $0.061(3)$ & $0.043(3)$ & $0.037(2)$ & $0.015(2)$ & $0.006(2)$ & $0.001(2)$ \\
\hline $\mathrm{C} 5$ & $0.043(2)$ & $0.038(2)$ & $0.0270(19)$ & $-0.0022(17)$ & $0.0093(17)$ & $-0.0014(18)$ \\
\hline $\mathrm{C} 6$ & $0.062(3)$ & $0.049(3)$ & $0.0244(19)$ & $-0.0012(18)$ & $0.0066(19)$ & $-0.009(2)$ \\
\hline C7 & $0.035(2)$ & $0.032(2)$ & $0.030(2)$ & $0.0014(16)$ & $0.0008(15)$ & $0.0041(17)$ \\
\hline $\mathrm{C} 8$ & $0.041(2)$ & $0.027(2)$ & $0.034(2)$ & $-0.0015(16)$ & $0.0044(17)$ & $-0.0059(18)$ \\
\hline $\mathrm{C} 9$ & $0.0320(18)$ & $0.0274(19)$ & $0.0253(17)$ & $0.0025(14)$ & $-0.0005(14)$ & $-0.0014(15)$ \\
\hline $\mathrm{C} 10$ & $0.044(2)$ & $0.039(3)$ & $0.039(2)$ & $-0.0059(19)$ & $0.0063(19)$ & $-0.001(2)$ \\
\hline $\mathrm{C} 11$ & $0.050(3)$ & $0.029(2)$ & $0.036(2)$ & $0.0034(17)$ & $0.0100(19)$ & $-0.0042(19)$ \\
\hline $\mathrm{C} 12$ & $0.036(2)$ & $0.037(2)$ & 0.0298 (19) & $0.0048(17)$ & $-0.0052(16)$ & $-0.0088(18)$ \\
\hline $\mathrm{C} 13$ & $0.033(2)$ & $0.037(2)$ & $0.035(2)$ & $0.0049(18)$ & $-0.0052(16)$ & $0.0016(17)$ \\
\hline $\mathrm{C} 14$ & $0.0288(18)$ & 0.0281 (19) & $0.033(2)$ & $0.0039(16)$ & $0.0040(15)$ & $-0.0016(15)$ \\
\hline C15 & $0.046(3)$ & $0.056(3)$ & $0.040(2)$ & $-0.003(2)$ & $-0.014(2)$ & $-0.002(2)$ \\
\hline C16 & $0.040(2)$ & $0.033(2)$ & $0.050(3)$ & $-0.002(2)$ & $0.005(2)$ & $0.0022(19)$ \\
\hline
\end{tabular}


Table S6. Hydrogen bond distances [A] and angles [deg] for 1.

\begin{tabular}{lllllcl}
\hline D & H & A & DH & HA & DA & DHA \\
\hline N2 & H2D & O4 & $0.90(5)$ & $2.55(5)$ & $3.159(5)$ & $126(4)$ \\
N2 & H2D & F10 & $0.90(5)$ & $2.59(6)$ & $3.467(5)$ & $166(4)$ \\
N2 & H2C & O2 & $0.87(6)$ & $2.31(6)$ & $3.152(5)$ & $165(5)$ \\
N2 & H2C & F6 & $0.87(6)$ & $2.64(6)$ & $3.275(5)$ & $131(5)$
\end{tabular}


Table s7. Crystal data and structure refinement for 4.

\begin{tabular}{|c|c|}
\hline Empirical formula & C18 H22 Cd F12 N2 O4 \\
\hline Formula weight & 670.78 \\
\hline Temperature & $153(2) \mathrm{K}$ \\
\hline Wavelength & $0.71069 \AA$ \\
\hline Crystal system, space group & Monoclinic, $\quad$ P2 (1)/n \\
\hline Unit cell dimensions & $\begin{array}{lll}\mathrm{a}=9.1913(6) & \AA & \alpha=90.0^{\circ} \\
\mathrm{b}=17.2805(11) & \AA & \beta=94.466^{\circ}(1) \\
\mathrm{c}=16.1356(11) & \AA & \gamma=90.0^{\circ}\end{array}$ \\
\hline Volume & $2555.0(3) \AA ̊ 3$ \\
\hline Z, Calculated density & 4, $\quad 1.744 \mathrm{Mg} / \mathrm{m} 3$ \\
\hline Absorption coefficient & $0.969 \mathrm{~mm}-1$ \\
\hline$F(000)$ & 1328 \\
\hline Crystal size & $0.10 \times 0.28 \times 0.30 \mathrm{~mm}$ \\
\hline Theta range for data collection & 1.73 to $28.99^{\circ}$ \\
\hline Limiting indices & $-12<=\mathrm{h}<=12, \quad-22<=\mathrm{k}<=23, \quad-21<=1<=21$ \\
\hline Reflections collected / unique & $23439 / 6280 \quad\left(R_{\text {int }}=0.0422\right)$ \\
\hline Completeness to theta $=28.99$ & $92.5 \div$ \\
\hline Absorption correction & integration \\
\hline Max. and min. transmission & 0.9149 and 0.7297 \\
\hline Refinement method & Full-matrix least-squares on $\mathrm{F}^{2}$ \\
\hline Data / restraints / parameters & $6280 / 0 / 330$ \\
\hline Goodness-of-fit on $\mathrm{F}^{\wedge} 2$ & 1.027 \\
\hline Final $R$ indices [I>2sigma $(I)]$ & $R 1=0.0630, w R 2=0.1830$ \\
\hline R indices (all data) & $\mathrm{R} 1=0.0817, \quad \mathrm{wR} 2=0.2069$ \\
\hline Largest diff. peak and hole & 1.715 and -0.971 e- $/ \AA-3$ \\
\hline
\end{tabular}


Table S8. Atomic coordinates and equivalent isotropic displacement parameters $\left(A^{\wedge} 2 \times 10^{\wedge} 3\right)$ for 4 .

$\mathrm{U}(\mathrm{eq})$ is defined as one third of the trace of the orthogonalized Uij tensor.

\begin{tabular}{|c|c|c|c|c|}
\hline & $\mathrm{x}$ & $\mathrm{Y}$ & $z$ & $\mathrm{U}(\mathrm{eq})$ \\
\hline $\mathrm{Cd} 1$ & $0.57035(4)$ & $0.207234(19)$ & $0.67308(2)$ & $0.04992(17)$ \\
\hline F1 & $0.9485(5)$ & $0.0109(3)$ & $0.7006(3)$ & $0.1081(16)$ \\
\hline $\mathrm{F} 2$ & $0.7760(6)$ & $-0.0616(3)$ & $0.6706(4)$ & $0.1159(18)$ \\
\hline F3 & $0.9494(12)$ & $-0.0248(6)$ & $0.5846(5)$ & $0.0821(16)$ \\
\hline F3B & $0.8951(15)$ & $-0.0497(8)$ & $0.5738(6)$ & $0.0821(16)$ \\
\hline F4 & $0.5011(5)$ & $0.1243(3)$ & $0.3707(3)$ & $0.1024(15)$ \\
\hline F5 & $0.7316(5)$ & $0.1146(2)$ & $0.3609(2)$ & $0.0861(12)$ \\
\hline F6 & $0.6368(5)$ & $0.2253(2)$ & $0.3804(3)$ & $0.0859(12)$ \\
\hline F7 & $0.3015(5)$ & $0.4273(3)$ & $0.5485(3)$ & $0.1039(15)$ \\
\hline F8 & $0.4421(5)$ & $0.5172(2)$ & $0.5963(3)$ & $0.0967(14)$ \\
\hline F9 & $0.3058(4)$ & $0.4563(2)$ & $0.6759(3)$ & $0.0854(11)$ \\
\hline F10 & $1.0202(8)$ & $0.3538(4)$ & $0.6762(4)$ & $0.0870(10)$ \\
\hline F11 & $0.9336(7)$ & $0.4573(4)$ & $0.6162(5)$ & $0.0870(10)$ \\
\hline $\mathrm{F} 12$ & $0.9552(8)$ & $0.3541(4)$ & $0.5469(4)$ & $0.0870(10)$ \\
\hline F13 & $0.9597(14)$ & $0.4405(8)$ & $0.6746(9)$ & $0.0870(10)$ \\
\hline F14 & $0.9085(15)$ & $0.4507(8)$ & $0.5652(9)$ & $0.0870(10)$ \\
\hline F15 & $0.9914(15)$ & $0.3387(9)$ & $0.5747(9)$ & $0.0870(10)$ \\
\hline 01 & $0.7053(4)$ & $0.0960(2)$ & $0.6710(2)$ & $0.0607(9)$ \\
\hline $\mathrm{O} 2$ & $0.5724(5)$ & $0.1866(3)$ & $0.5330(3)$ & $0.0641(10)$ \\
\hline 03 & $0.4574(4)$ & $0.3252(2)$ & $0.6597(3)$ & $0.0624(10)$ \\
\hline ○4 & $0.7654(4)$ & $0.2864(2)$ & $0.6635(3)$ & $0.0642(10)$ \\
\hline $\mathrm{N1}$ & $0.3385(6)$ & $0.1514(3)$ & $0.6728(4)$ & $0.0681(13)$ \\
\hline N2 & $0.5495(7)$ & $0.1903(3)$ & $0.8153(3)$ & $0.0706(13)$ \\
\hline $\mathrm{C} 1$ & $0.7570(5)$ & $0.0639(3)$ & $0.6112(4)$ & $0.0539(11)$ \\
\hline $\mathrm{C} 2$ & $0.7389(6)$ & $0.0813(3)$ & $0.5273(3)$ & $0.0552(12)$ \\
\hline $\mathrm{H} 2$ & 0.7903 & 0.0514 & 0.4897 & 0.066 \\
\hline $\mathrm{C} 3$ & $0.6476(5)$ & $0.1413(3)$ & $0.4953(3)$ & $0.0501(11)$ \\
\hline $\mathrm{C} 4$ & $0.8528(7)$ & $-0.0074(4)$ & $0.6375(5)$ & $0.0735(17)$ \\
\hline $\mathrm{C} 5$ & $0.6322(7)$ & $0.1512(4)$ & $0.4010(4)$ & $0.0645(14)$ \\
\hline $\mathrm{C} 6$ & $0.5070(5)$ & $0.3862(3)$ & $0.6329(3)$ & $0.0484(10)$ \\
\hline $\mathrm{C} 7$ & $0.6498(6)$ & $0.4051(3)$ & $0.6199(3)$ & $0.0512(11)$ \\
\hline $\mathrm{H} 7$ & 0.6699 & 0.4551 & 0.5991 & 0.061 \\
\hline C8 & $0.7644(6)$ & $0.3541(3)$ & $0.6359(3)$ & $0.0522(11)$ \\
\hline C9 & $0.3885(6)$ & $0.4482(3)$ & $0.6138(4)$ & $0.0602(13)$ \\
\hline $\mathrm{C} 10$ & $0.9194(7)$ & $0.3826(3)$ & $0.6212(4)$ & $0.0695(16)$ \\
\hline $\mathrm{C} 11$ & $0.2909(8)$ & $0.1643(5)$ & $0.7527(5)$ & $0.086(2)$ \\
\hline $\mathrm{H} 11 \mathrm{~A}$ & 0.2036 & 0.1324 & 0.7604 & 0.103 \\
\hline $\mathrm{H} 11 \mathrm{~B}$ & 0.2640 & 0.2194 & 0.7586 & 0.103 \\
\hline $\mathrm{C} 12$ & $0.4132(8)$ & $0.1431(5)$ & $0.8183(4)$ & $0.0765(18)$ \\
\hline $\mathrm{H} 12 \mathrm{~A}$ & 0.3763 & 0.1491 & 0.8740 & 0.092 \\
\hline $\mathrm{H} 12 \mathrm{~B}$ & 0.4383 & 0.0879 & 0.8114 & 0.092 \\
\hline $\mathrm{C} 13$ & $0.2300(8)$ & $0.1927(4)$ & $0.6044(6)$ & $0.084(2)$ \\
\hline H13A & 0.2294 & 0.2488 & 0.6166 & 0.101 \\
\hline $\mathrm{H} 13 \mathrm{~B}$ & 0.2696 & 0.1861 & 0.5496 & 0.101 \\
\hline $\mathrm{C} 14$ & $0.0780(9)$ & $0.1650(5)$ & $0.5979(6)$ & $0.102(3)$ \\
\hline $\mathrm{H} 14 \mathrm{~A}$ & 0.0726 & 0.1149 & 0.5695 & 0.153 \\
\hline
\end{tabular}




\begin{tabular}{lllll} 
H14B & 0.0159 & 0.2025 & 0.5663 & 0.153 \\
H14C & 0.0443 & 0.1592 & 0.6538 & 0.153 \\
C15 & $0.3466(7)$ & $0.0684(3)$ & $0.6502(5)$ & $0.0776(18)$ \\
H15A & 0.4249 & 0.0434 & 0.6852 & 0.116 \\
H15B & 0.3668 & 0.0637 & 0.5917 & 0.116 \\
H15C & 0.2534 & 0.0432 & 0.6588 & 0.116 \\
C16 & $0.6715(9)$ & $0.1506(4)$ & $0.8601(4)$ & $0.0812(19)$ \\
H16A & 0.6750 & 0.0970 & 0.8388 & 0.097 \\
H16B & 0.6518 & 0.1476 & 0.9194 & 0.097 \\
C17 & $0.8174(9)$ & $0.1861(6)$ & $0.8547(6)$ & $0.098(2)$ \\
H17A & 0.8403 & 0.1881 & 0.7965 & 0.147 \\
H17B & 0.8912 & 0.1550 & 0.8867 & 0.147 \\
H17C & 0.8172 & 0.2387 & 0.8775 & 0.147 \\
C18 & $0.5312(13)$ & $0.2667(4)$ & $0.8529(5)$ & $0.105(3)$ \\
H18A & 0.5239 & 0.2609 & 0.9128 & 0.158 \\
H18B & 0.4420 & 0.2910 & 0.8278 & 0.158 \\
H18C & 0.6155 & 0.2993 & 0.8431 & 0.158 \\
& & & & \\
\hline
\end{tabular}


Table S9. Bond lengths [A] and angles [deg] for 4.

\begin{tabular}{|c|c|c|}
\hline $\mathrm{Cd} 1$ & O4 & $2.270(4)$ \\
\hline $\operatorname{Cd} 1$ & $\mathrm{O} 1$ & $2.289(4)$ \\
\hline $\mathrm{Cd} 1$ & 03 & $2.290(4)$ \\
\hline $\mathrm{Cd} 1$ & $\mathrm{O} 2$ & $2.290(4)$ \\
\hline $\mathrm{Cd} 1$ & N2 & $2.337(6)$ \\
\hline $\mathrm{Cd} 1$ & N1 & $2.339(5)$ \\
\hline F1 & $\mathrm{C} 4$ & $1.330(9)$ \\
\hline $\mathrm{F} 2$ & $\mathrm{C} 4$ & $1.310(9)$ \\
\hline F3 & F3B & $0.670(11)$ \\
\hline F3 & $\mathrm{C} 4$ & $1.313(10)$ \\
\hline F3B & $\mathrm{C} 4$ & $1.343(13)$ \\
\hline F4 & $\mathrm{C} 5$ & $1.348(8)$ \\
\hline F5 & C5 & $1.321(7)$ \\
\hline F6 & C5 & $1.324(7)$ \\
\hline F7 & C9 & $1.323(7)$ \\
\hline F8 & C9 & $1.330(7)$ \\
\hline F9 & C9 & $1.312(7)$ \\
\hline F10 & $\mathrm{C} 10$ & $1.328(10)$ \\
\hline F10 & F13 & $1.597(15)$ \\
\hline F10 & F15 & $1.659(15)$ \\
\hline F11 & F14 & $0.845(13)$ \\
\hline F11 & F13 & $0.998(13)$ \\
\hline F11 & $\mathrm{C} 10$ & $1.301(9)$ \\
\hline F12 & F15 & $0.599(14)$ \\
\hline F12 & $\mathrm{C} 10$ & $1.360(10)$ \\
\hline F12 & F14 & $1.754(16)$ \\
\hline F13 & $\mathrm{C} 10$ & $1.354(15)$ \\
\hline F14 & $\mathrm{C} 10$ & $1.482(15)$ \\
\hline F15 & $\mathrm{C} 10$ & $1.286(15)$ \\
\hline O1 & $\mathrm{C} 1$ & $1.240(7)$ \\
\hline $\mathrm{O} 2$ & $\mathrm{C} 3$ & $1.236(6)$ \\
\hline $\mathrm{O3}$ & $\mathrm{C} 6$ & $1.241(6)$ \\
\hline 04 & $\mathrm{C} 8$ & $1.250(6)$ \\
\hline N1 & $\mathrm{C} 11$ & $1.410(9)$ \\
\hline N1 & C15 & $1.484(7)$ \\
\hline N1 & C13 & $1.597(10)$ \\
\hline N2 & $\mathrm{C} 16$ & $1.457(9)$ \\
\hline N2 & $\mathrm{C} 18$ & $1.468(8)$ \\
\hline N2 & $\mathrm{C} 12$ & $1.498(9)$ \\
\hline $\mathrm{C} 1$ & $\mathrm{C} 2$ & $1.384(8)$ \\
\hline $\mathrm{C} 1$ & $\mathrm{C} 4$ & $1.555(8)$ \\
\hline $\mathrm{C} 2$ & $\mathrm{C} 3$ & $1.406(7)$ \\
\hline $\mathrm{C} 3$ & C5 & $1.527(8)$ \\
\hline $\mathrm{C} 6$ & $\mathrm{C} 7$ & $1.384(7)$ \\
\hline $\mathrm{C} 6$ & C9 & $1.541(7)$ \\
\hline $\mathrm{C} 7$ & C8 & $1.382(7)$ \\
\hline $\mathrm{C} 8$ & $\mathrm{C} 10$ & $1.543(8)$ \\
\hline C11 & $\mathrm{C} 12$ & $1.528(11)$ \\
\hline C13 & $\mathrm{C} 14$ & 1.473 (11) \\
\hline C16 & $\mathrm{C} 17$ & $1.483(11)$ \\
\hline O4 & Cal O1 & $94.21(14)$ \\
\hline & Ca1 O3 & $79.14(13)$ \\
\hline
\end{tabular}




\begin{tabular}{|c|c|c|}
\hline $\mathrm{O} 1$ & $\mathrm{Cd} 1$ & 03 \\
\hline O4 & $\mathrm{Cd} 1$ & $\mathrm{O} 2$ \\
\hline 01 & $\mathrm{Cd} 1$ & $\mathrm{O} 2$ \\
\hline 3 & $\mathrm{Cd} 1$ & $\mathrm{O} 2$ \\
\hline O4 & $\mathrm{Cd} 1$ & N2 \\
\hline 1 & $\mathrm{Cd} 1$ & N2 \\
\hline 3 & $\mathrm{Cd} 1$ & $\mathrm{~N} 2$ \\
\hline 2 & $\mathrm{Cd} 1$ & N2 \\
\hline 4 & $\mathrm{Cd} 1$ & $\mathrm{~N} 1$ \\
\hline 1 & $\mathrm{Cd} 1$ & $\mathrm{~N} 1$ \\
\hline 3 & $\mathrm{CdI}$ & $\mathrm{N} 1$ \\
\hline 2 & $\mathrm{Cd} 1$ & N1 \\
\hline 2 & $\mathrm{Cd} 1$ & N1 \\
\hline $3 B$ & F3 & $\mathrm{C} 4$ \\
\hline F3 & F3B & $\mathrm{C} 4$ \\
\hline 10 & F10 & F13 \\
\hline 10 & F10 & F15 \\
\hline 13 & F10 & F15 \\
\hline 14 & $F 11$ & F13 \\
\hline F14 & F11 & $\mathrm{C} 10$ \\
\hline 13 & $F 11$ & $\mathrm{C} 10$ \\
\hline F15 & F12 & $\mathrm{C} 10$ \\
\hline 15 & F12 & F14 \\
\hline $\mathrm{C} 10$ & F12 & F14 \\
\hline 11 & F13 & $\mathrm{C} 10$ \\
\hline 11 & F13 & F10 \\
\hline 10 & F13 & F10 \\
\hline 11 & F14 & $\mathrm{C} 10$ \\
\hline 11 & F14 & $\mathrm{F} 12$ \\
\hline $\mathrm{C} 10$ & F14 & F12 \\
\hline 12 & F15 & $\mathrm{C} 10$ \\
\hline 12 & F15 & F10 \\
\hline $\mathrm{C} 10$ & F15 & F10 \\
\hline $\mathrm{C} 1$ & $\mathrm{O} 1$ & $\mathrm{Cd} 1$ \\
\hline C3 & $\mathrm{O} 2$ & $\mathrm{Cd} 1$ \\
\hline C6 & 03 & Cal \\
\hline $\mathrm{C} 8$ & O4 & $\mathrm{Cd} 1$ \\
\hline C11 & N1 & 15 \\
\hline $\mathrm{C} 11$ & N1 & C13 \\
\hline C15 & N1 & 13 \\
\hline C11 & N1 & $\mathrm{Cd} 1$ \\
\hline C15 & N1 & Cal \\
\hline C13 & N1 & $\mathrm{Cal}$ \\
\hline C16 & N2 & 18 \\
\hline C16 & N2 & $\mathrm{C} 12$ \\
\hline C18 & N2 & $\mathrm{C} 12$ \\
\hline C16 & N2 & $\mathrm{Cd} 1$ \\
\hline C18 & N2 & $\mathrm{Cd} 1$ \\
\hline C12 & N2 & $\mathrm{Cal}$ \\
\hline O1 & $\mathrm{C} 1$ & $\mathrm{C} 2$ \\
\hline 01 & $\mathrm{C} 1$ & $\mathrm{C} 4$ \\
\hline $\mathrm{C} 2$ & $\mathrm{C} 1$ & $\mathrm{C} 4$ \\
\hline $\mathrm{C} 1$ & $\mathrm{C} 2$ & C3 \\
\hline $\mathrm{O} 2$ & C3 & $\mathrm{C} 2$ \\
\hline $\mathrm{O} 2$ & C3 & C5 \\
\hline $\mathrm{C} 2$ & C3 & C5 \\
\hline F3 & $\mathrm{C} 4$ & F2 \\
\hline
\end{tabular}

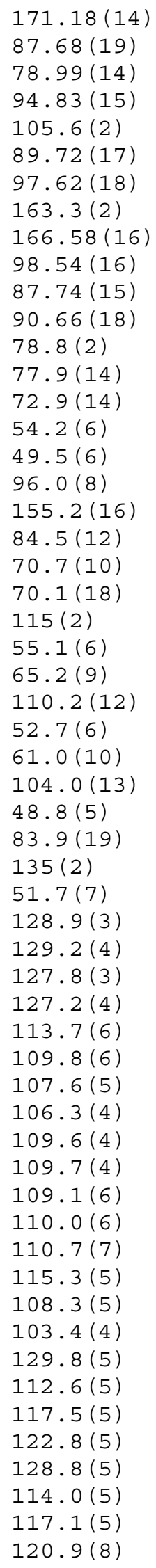




$\begin{array}{lll}\text { F3 } & \text { C4 } & \text { F1 } \\ \text { F2 } & \text { C4 } & \text { F1 } \\ \text { F3 } & \text { C4 } & \text { F3B } \\ \text { F2 } & \text { C4 } & \text { F3B } \\ \text { F1 } & \text { C4 } & \text { F3B } \\ \text { F3 } & \text { C4 } & \text { C1 } \\ \text { F2 } & \text { C4 } & \text { C1 } \\ \text { F1 } & \text { C4 } & \text { C1 } \\ \text { F3B } & \text { C4 } & \text { C1 } \\ \text { F5 } & \text { C5 } & \text { F6 } \\ \text { F5 } & \text { C5 } & \text { F4 } \\ \text { F6 } & \text { C5 } & \text { F4 } \\ \text { F5 } & \text { C5 } & \text { C3 } \\ \text { F6 } & \text { C5 } & \text { C3 } \\ \text { F4 } & \text { C5 } & \text { C3 } \\ \text { O3 } & \text { C6 } & \text { C7 } \\ \text { O3 } & \text { C6 } & \text { C9 } \\ \text { C7 } & \text { C6 } & \text { C9 } \\ \text { C8 } & \text { C7 } & \text { C6 } \\ \text { O4 } & \text { C8 } & \text { C7 } \\ \text { O4 } & \text { C8 } & \text { C10 } \\ \text { C7 } & \text { C8 } & \text { C10 } \\ \text { F9 } & \text { C9 } & \text { F7 } \\ \text { F9 } & \text { C9 } & \text { F8 } \\ \text { F7 } & \text { C9 } & \text { F8 } \\ \text { F9 } & \text { C9 } & \text { C6 } \\ \text { F7 } & \text { C9 } & \text { C6 } \\ \text { F8 } & \text { C9 } & \text { C6 } \\ \text { F15 } & \text { C10 } & \text { F11 } \\ \text { F15 } & \text { C10 } & \text { F10 } \\ \text { F11 } & \text { C10 } & \text { F10 } \\ \text { F15 } & \text { C10 } & \text { F12 } \\ \text { F11 } & \text { C10 } & \text { F12 } \\ \text { F10 } & \text { C10 } & \text { F12 } \\ \text { F15 } & \text { C10 } & \text { F13 } \\ \text { F11 } & \text { C10 } & \text { F13 } \\ \text { F10 } & \text { C10 } & \text { F13 } \\ \text { F12 } & \text { C10 } & \text { F13 } \\ \text { F15 } & \text { C10 } & \text { F14 } \\ \text { F11 } & \text { C10 } & \text { F14 } \\ \text { F10 } & \text { C10 } & \text { F14 } \\ \text { F12 } & \text { C10 } & \text { F14 } \\ \text { F13 } & \text { C10 } & \text { F14 } \\ \text { F15 } & \text { C10 } & \text { C8 } \\ \text { F11 } & \text { C10 } & \text { C8 } \\ \text { F10 } & \text { C10 } & \text { C8 } \\ \text { F12 } & \text { C10 } & \text { C8 } \\ \text { F13 } & \text { C10 } & \text { C8 } \\ \text { F14 } & \text { C10 } & \text { C8 } \\ \text { N1 } & \text { C11 } & \text { C12 } \\ \text { N2 } & \text { C12 } & \text { C11 } \\ \text { C14 } & \text { C13 } & \text { N1 } \\ \text { N2 } & \text { C16 } & \text { C17 }\end{array}$

96.4(7)

$101.8(6)$

$29.2(5)$

$96.9(8)$

$120.1(8)$

$113.6(6)$

$111.5(5)$

$110.3(6)$

$114.4(7)$

$107.6(5)$

$106.9(5)$

$106.7(6)$

$114.9(5)$

$110.9(5)$

$109.5(5)$

$129.2(5)$

$112.8(4)$

$118.1(4)$

$122.6(5)$

$130.3(5)$

$111.9(5)$

$117.8(5)$

106.7 (5)

$108.2(5)$

$106.6(5)$

$111.3(5)$

$110.1(5)$

$113.4(5)$

$119.3(9)$

$78.8(8)$

$110.1(7)$

$26.0(6)$

$105.8(7)$

$104.4(6)$

$132.8(10)$

$44.1(6)$

$73.1(8)$

$139.1(8)$

$97.2(9)$

$34.6(5)$

$135.6(8)$

$76.1(7)$

$78.7(8)$

$115.1(8)$

$115.2(6)$

$112.5(6)$

$108.0(6)$

$110.4(7)$

$109.2(7)$

$109.3(6)$

$114.8(6)$

$116.3(7)$

$116.2(7)$ 
Table S10. Anisotropic displacement parameters ( $\left.A^{\wedge} 2\right)$ for 4. The anisotropic displacement factor exponent takes the form: $-2 \mathrm{pi}^{\wedge} 2$ [ h^2 $\mathrm{a}^{{ }^{\wedge} 2} \mathrm{U11}+\ldots+2 \mathrm{hk} \mathrm{a}^{*} \mathrm{~b} * \mathrm{U} 12$ ]

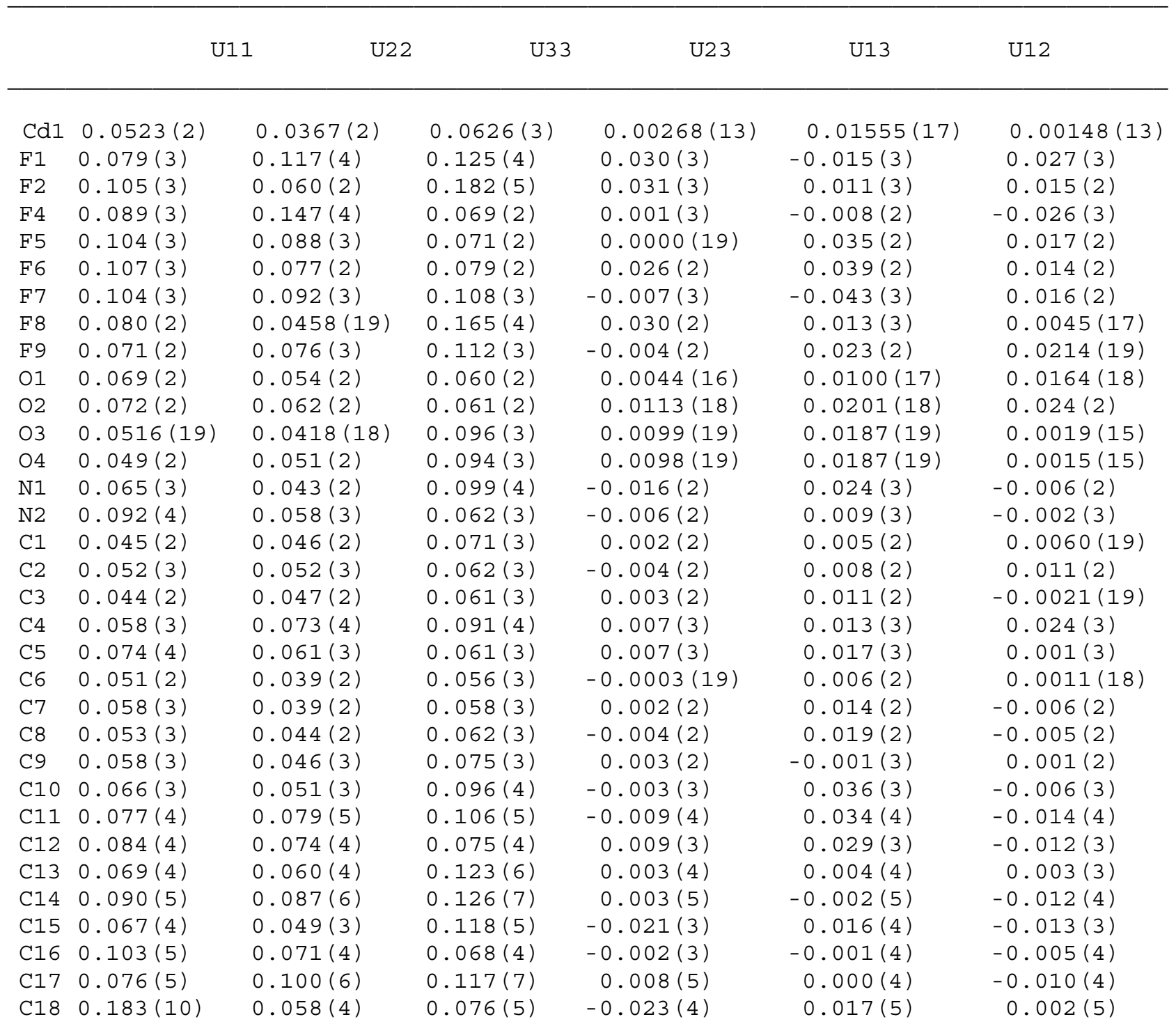


Figure S1. Packing diagram for 4. Non-hydrogen bonded hydrogen and disordered fluorine atoms are ommited for clarity.

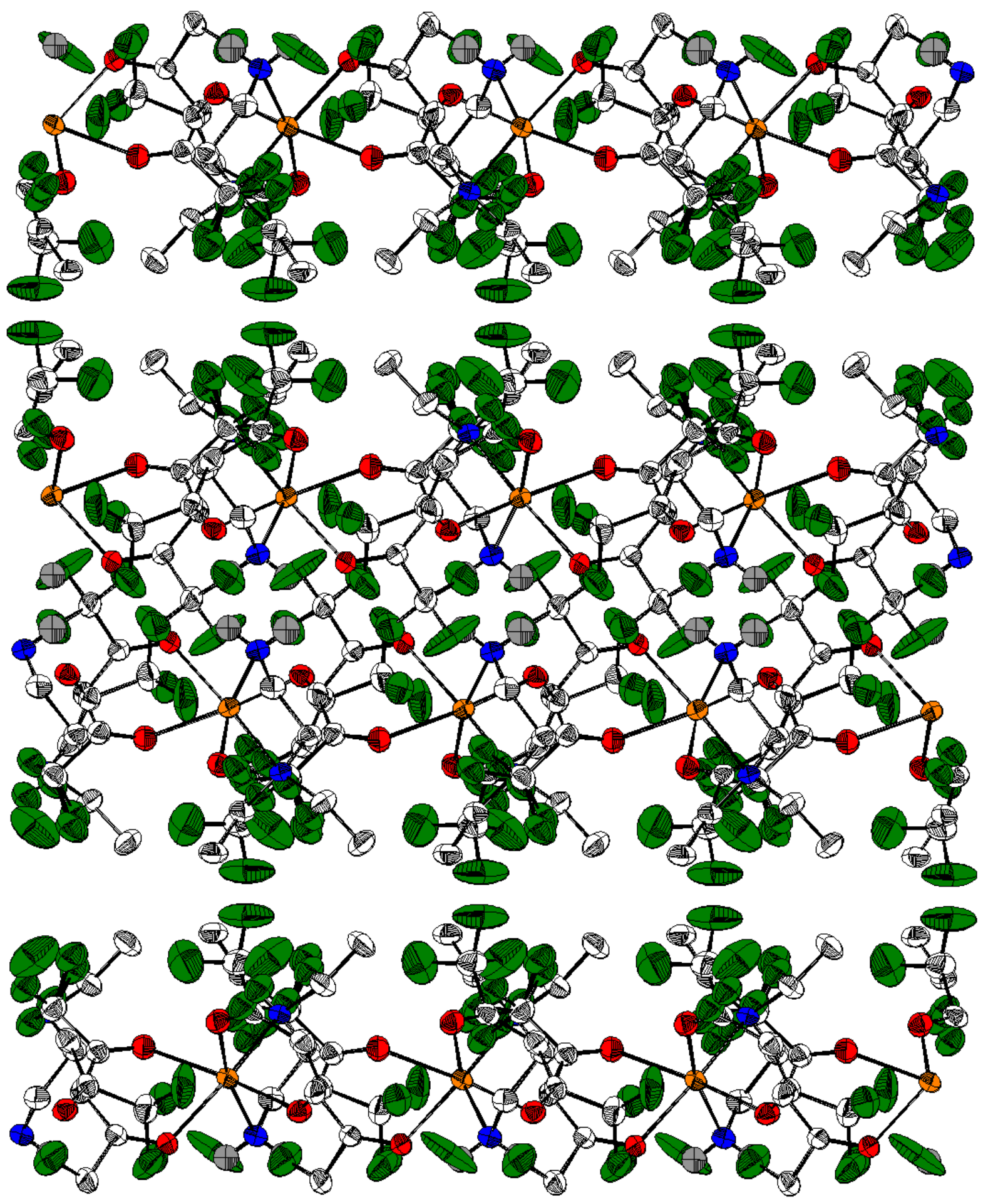


(A)

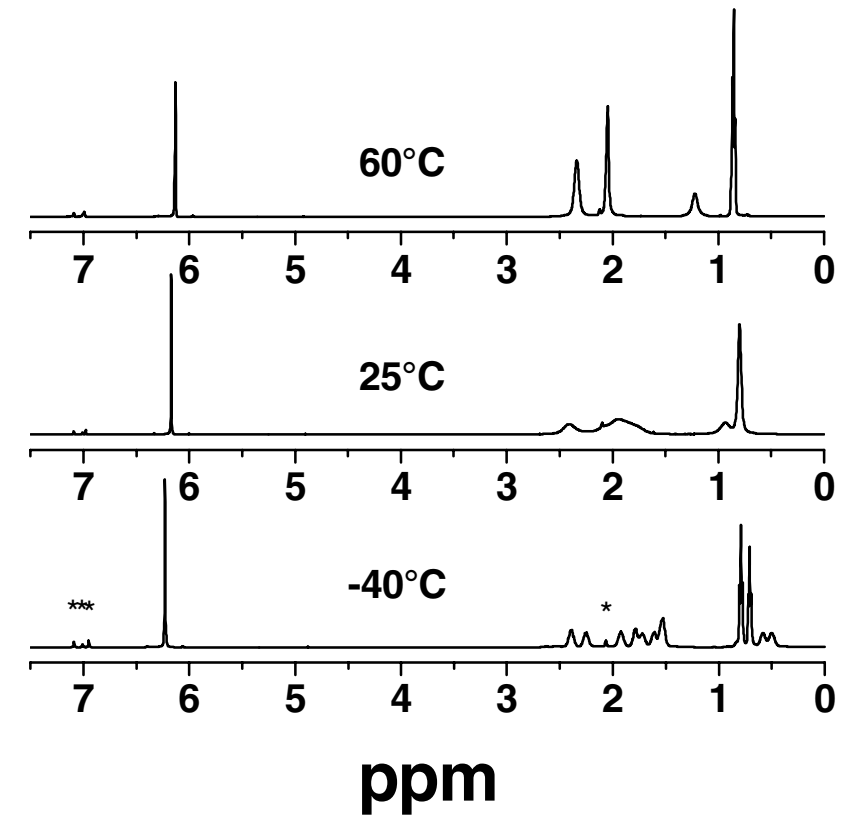

(B)
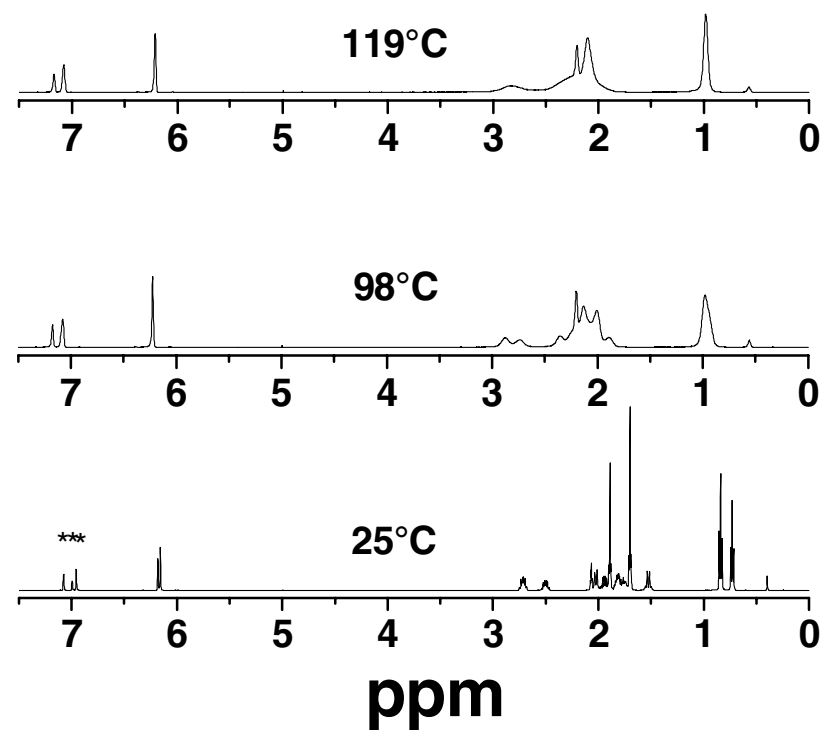

Figure s2. Variable-temperature ${ }^{1} \mathrm{H}$ NMR of complex 2 (A) and complex 4 (B) in toluene- $d_{8}$ at $500 \mathrm{MHz}$. * Denote solvent resonances. 